\title{
Evaluation Optimal Friction Factor Correlation in Turbulent Pipe Flow by Genetic Algorithm
}

\author{
Qais Abid Yousif ${ }^{1}$, Omar Rafae Alomar ${ }^{1}$, Ibrahim Atiya Mohamed ${ }^{1}$, Majid Kh. Najm ${ }^{1}$ \\ (kaisyusuf@ntu.edu.iq, omar.alomar@ntu.edu.iq, ibams_1962@ ntu.edu.iq, dr.majid.najim@ntu.edu.iq) \\ ${ }^{1}$ Northern Technical University, Engineering Technical College of Mosul, Cultural Group Street, \\ Mosul, Iraq
}

\begin{abstract}
The prediction accuracies of friction factor correlations in turbulent pipe flow have remained unsatisfactory due to Colebrook equation is characterized as an implicit correlation. Thus, this works deals with numerical simulation for optimization the correlation of friction factor $\left(f_{D}\right)$ in turbulent pipe flow. Genetic Algorithms (GAs) method has been used to evaluate the accuracy of six most used explicit models as an alternative to the Colebrook equation. The $f_{D}$ has been estimated for higher ranges of Reynolds Number $(\operatorname{Re})$ and the relative roughness of pipe $(\varepsilon / D)$. The evaluation process has been implemented through comparing the percentage of differences between the values of $f_{D}$ obtained using those correlations with that obtained using Colebrook equation. The optimized results clearly show that the Model-1 and Model-5 provide the lowest percentage of difference as compared to the other explicit models. Results indicated that GAs has succeeded in reducing the computational time by eliminating the iterative process.
\end{abstract}

Keywords: Colebrook equation, friction factor, genetic algorithms.

\section{Introduction}

Despite the great scientific progress that has been made in computer systems and the multiplicity of mathematical methods and programs developed, but still, the Colebrook equation is used in the evaluation of the coefficient of friction [1]. Although the accuracy of Colebrook equation is debatable, it is occasionally necessary to achieve an accurate solution of this equation extremely important for the scientific calculations and frequently for comparisons [2]. Various techniques have been utilized either to compute or to appraise the friction factor precisely. The greater part of recent efforts has been utilized Genetic Algorithms (GAs) and Artificial Neural Network method (ANN) for computing friction factor [3]. However, the use of GAs in the area of heat transfer is very newfangled. This is perhaps due to the computational time needed to reach the final solutions is too long. Recently, the GAs has been increasingly used in heat transfer problems. In 1994, Queipo et al. [4] have expected that the heat transfer community will witness considerable attention for involving new methods such as techniques (i.e. GAs) in many intricate thermo-science problems that confessing optimization. One that has helped to facilitate the use of these exciting technologies in the development of high-performance computing devices, which has increased interest in their use in the field of heat transfer. The 
ANN and GAs have been affirmed and showed the key points of interest to boost traditional techniques for associating experimental data [5 - 13].

Chow et al. [5] used a combination between ANN and AGs to find the optimal costs of achieving the economical operation process of the direct-fired double-effect absorption chiller system. The results demonstrated that this objective can be accomplished by applying these two advanced approaches. Xie et al. [6] applied the GAs to optimize the structure sizes of fin-andtube heat exchangers through finding the minimal entire weight and minimal annual cost. The results show that the GAs has a comprehensive ability for accomplishing minimum weight and cost of fin-and-tube heat exchangers that have various and particular applications. Later, Xie et al. [7] implemented the optimization study on the e-NTU by using GAs to find a minimal volume that corresponds with minimal annual cost of design a plate - fin type compact heat exchanger. They demonstrated that GAs is an effective approach for optimization and hence, it can furnish a robust potential of auto-search and mixed optimization in the optimization of heat exchangers in comparison with the conventional methods. Özger and Yıldırım [8] elaborated a neuro - fuzzy approach in order to find an accurate correlation that connects pipe roughness and Reynolds number with friction factor due to that the majority of the available data are uncertainties and inaccurate. They have observed that the friction factor can be precisely evaluated. Brkić $[9,10]$ developed a simple and accurate approximation for the Colebrook equation based on the solution of Lambert $\mathrm{W}$-function. This approximation can be used to estimate the friction factor in case of higher Reynolds number and relative roughness. Besarati et al. [11] developed a simple and accurate explicit equation for the friction factor by improving an artificial neural network approach by using multi-objective of GAs. The proposed approach can be used for different ranges of Reynolds number and relative roughness. Lin et al. [12] employed the GAs to enhance the efficiency of heating, ventilation, and air conditioning (HVAC) systems using fan coil unit (FCU) for controlling temperature and energy conservation in chilled water systems. In order to exhibit the efficiency of the advised HVAC system, they have performed field experiments and the results show that the GAs has been succeeded for determining the frequency of the operating pump-related with minimal energy consumption per refrigeration ton and accomplished the purpose of controlling the energy conservation. In their study, the goal of improvement has been achieved.

A careful look into the literature review related to GAs in the field of heat transfer, it is possible to guess the magnitude of the challenges and future possibilities. Great attention has been given to this topic particularly after 2003 [3]. Although the GAs has some restrictions, it appears to be a favourable and attainable replacement for designing and optimizing the thermal systems such as heat exchangers, heat and fluid flow networks, fins, porous media, heat sinks, etc. On the other hand, it has been pointed out that the ANN method has indicated to be helpful for approximation the space of the design in many problems [6-13]. Several studies employed the GAs for evaluating the friction factor [14-18]. However, the results show that the advanced approach can be utilized with either a suitable dataset or suitable equations in order to obtain an appropriate and accurate solution.

Although Colebrook equation is characterized as an implicit correlation for estimating the value of friction factor, it is still used until now for calculation the friction factor inside pipes under turbulent flow condition regardless of the existence of several explicit correlations that are developed to avoid the iterative process that emerges when using Colebrook equation. It is 
also clear from the literature review that most of the previous studies have been utilized GAs and ANN methods either to evaluate the friction factor (or frictional pressure drop) or to find the optimal design of a particular system with minimal size and annual cost. Nevertheless, these methods have been not used to evaluate the accuracy of the proposed explicit correlations in comparison with Colebrook equation. Therefore, the present study aims to use GAs for evaluation the accuracy of six most used explicit models as an alternative to the implicit Colebrook equation for calculation the Darcy friction factor $\left(f_{D}\right)$ inside a circular duct under turbulent flow condition. A single objective optimization based on GAs has been used. The purpose of using this algorithm is to avoid the iterative process and to reduce the computational time needed to obtain a solution when using Colebrook equation. The $f_{D}$ has been evaluated in terms of Reynolds number $(R e)$ and relative roughness $(\varepsilon / D)$ of the duct. The evaluation process has been performed through comparing the percentage difference of $f_{D}$ value obtained by using those six explicit Models with that obtained using Colebrook equation.

\section{Mathematical Model}

The flow resistance relies upon the flow rate, which is considered as the fundamental element in the determination of the friction factor. The flow resistance through pipes, is commonly expressed by the Darcy friction factor $\left(f_{D}\right)$. In Reality, the value of $f_{D}$ is not constant and hence, it depends on several parameters such as Reynolds number $(R e)$ and relative roughness $(\varepsilon / D)$. These two parameters are dimensionless. The $R e$ is also influenced by the mass flux, whereas $\varepsilon / D$ relies on the thickness of the flow (i.e., boundary layer) which is generated above the wall of the pipe [19]. In this study, $\varepsilon / D$ has been dealt as a geometric amount and hence, the value of this parameter should be constant. In general, $f_{D}$ has been estimated by using Colebrook equation [15, 16]. Although the Colebrook equation is implicit and needs to be iterated while finding the solutions of $f_{D}$ and hence, it requires longer computational time to reach final solutions. However, this equation is still used as a recognized criterion for estimating the $f_{D}$ inside pipes. The $f_{D}$ in Colebrook equation is defined in terms of $\operatorname{Re}$ and $\varepsilon / D$ as following:

$$
\frac{1}{\sqrt{f_{D}}}=-2 \log \left[\frac{\left(\frac{\varepsilon}{D}\right)}{3.71}+\frac{2.51}{R e \sqrt{f_{D}}}\right]
$$

Different strategies have been utilized while estimating the value of $f_{D}$ in Eq. (1) due to this equation has an implicit form and consequently, different explicit correlations (e.g., Eqs. (2) - (7)) have been developed with aims to obtain rid of iteration and in the meantime find the accurate solution of Eq. (1). The iterative solution obtained by using Eq. (1) has been supposed to be highly accurate and hence, the correlations that developed have different accuracies. Therefore, the evaluation process of the accuracy of the proposed explicit approximations that available in the literature in comparison with Colebrook equation (i.e., Eq. (1)) has been performed under turbulent flow conditions. The ranges of $R e$ and $\varepsilon / D$ have been considered to vary in wide ranges between 3000 and $10^{8}$ and between $10^{-5}$ and 0.1 , respectively. In the present study, six different models (correlations) have been considered and compared with Eq. (1). These correlations are set as follows: 
Model-1 according to the correlation [19]:

$$
f_{D}=0.25\left[\log \left\{\frac{(\varepsilon / D)}{3.7}-\left(\frac{5.02}{R e}\right) A\right\}\right]^{-2}
$$

where $A=\log \left(\frac{(\varepsilon / D)}{3.7}-\left(\frac{5.02}{R e}\right) B\right)$ and $B=\log \left(\frac{(\varepsilon / D)}{3.7}+\frac{13}{R e}\right)$

Model-2 according to the correlation [20]:

$$
f_{D}=\left(A-\frac{(B-A)^{2}}{C-2 B+A}\right)^{-2}
$$

where $A=-2 \log \left(\frac{(\varepsilon / D)}{3.7}+\frac{12}{R e}\right), B=-2 \log \left(\frac{(\varepsilon / D)}{3.7}+\frac{2.51 A}{R e}\right)$ and $C=-2 \log \left(\frac{(\varepsilon / D)}{3.7}+\frac{2.51 B}{R e}\right)$

Model-3 according to the correlation [21]:

$$
f_{D}=0.25\left[\log \left\{\frac{(\varepsilon / D)}{3.7065}-\left(\frac{5.0272}{R e}\right) A\right\}\right]^{-2}
$$

where $A=\log \left[\frac{(\varepsilon / D)}{3.827}-\left(\frac{4.567}{R e}\right) B\right]$ and $B=\log \left[\left\{\frac{(\varepsilon / D)}{7.7918}\right\}^{0.9924}+\left(\frac{5.3326}{208.815+R e}\right)^{0.9345}\right]$

Model-4 according to the correlation [22]:

$$
f_{D}=\left[A-\left\{\frac{A+2 \log (B / R e)}{1+(2.18 / B)}\right\}\right]^{-2}
$$

where $A=\frac{1.7825 \log R e-1.41}{1+1.32(\sqrt{\varepsilon / D})}$ and $B=\frac{\operatorname{Re}(\varepsilon / D)}{3.7}+2.51 A$

Model-5 according to the correlation [23]:

$$
f_{D}=0.25\left[\log \left\{\frac{0.4587 R e}{(A-0.31)^{B}}\right\}\right]^{-2}
$$

where $A=0.124 \operatorname{Re}(\varepsilon / D)+2.303 \log (0.4587 \operatorname{Re})$ and $B=\frac{A}{A+0.9633}$

Model-6 according to the correlation [24]:

$$
f_{D}=0.25\left[\log \left\{\frac{(\varepsilon / D)}{3.7106}-\left(\frac{5}{\Re}\right) A\right\}\right]^{-2}
$$

where $A=\log \left[\frac{(\varepsilon / D)}{3.8597}-\left(\frac{4.795}{R e}\right) B\right]$ and $B=\log \left[\left\{\frac{(\varepsilon / D)}{7.646}\right\}^{0.9685}+\left(\frac{4.9755}{206.2795+R e}\right)^{0.8759}\right]$

It can be noted here that Eq. (1) has an implicit form, whereas the correlations in Eqs. (2) - (7) have an explicit form. Therefore, Eq. (1) requires iteration process to obtain a solution, which is not the case for latter correlations. 


\section{Method of Solution}

In the present study, the single objective optimization based on Genetic Algorithms (GAs) has been used to evaluate the above correlations while determining $f_{D}$. The form of GAs can be easily created by using MATLAB software. The models presented in Eqs. (1) - (7) have been considered a fitness function. To find the optimal value of $f_{D}$, the GAs has been applied along with the individuals' operator for crossover based on fitness. The crossover recombines the genetic material of the selected parents, and then the mutation operator modifies the individuals that have been not selected for reproduction by randomly changing. The GAs tools used in this work are the optimization toolbox (version 9.4.0.813654) in MATLAB R2018a [25]. Firstly, the input datasets $(\operatorname{Re}$ and $\varepsilon / D)$ presented in the Colebrook equation (i.e., Eq. (1)) have been utilized in order to prepare the GAs forms and then, the friction factor has been anticipated by obtaining the GAs structure with a less relative error.

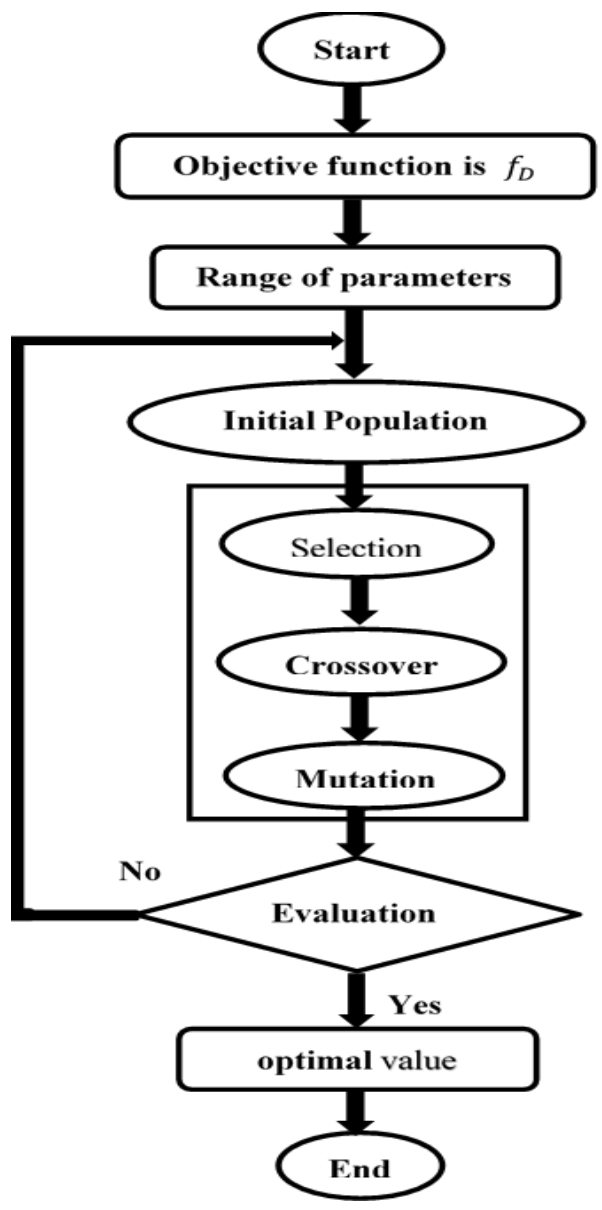

Fig. 1 The working process of the Gas 
For the purpose of comparison, the iterative solution of Eq. (1) has been firstly generated using the ranges values of $\operatorname{Re}$ and $\varepsilon / D$ and it has been initialized as the exact solution (i.e., reference solution). On the other hand, the solutions of the proposed correlations in Eqs. (2) (7) can be obtained without the iteration process due to their explicit form. The flowchart of the working process is shown in Fig. 1. The evolution starts with a random population of individuals, and then the initial population develops based on the selection, crossover and mutation operators with the objective function of $f_{D}$. This process is iterated until some criterion reaches an optimal value. Due to the stochastic nature of this method, the algorithm is stopped after 200 generations. Every fifty GAs runs, a uniformly random population has been started, where all the parameters values are equally represented in the initial population. Based on the random nature of the GAs, each optimization has been running five times. It can be noted here that the increase in the number of runs more than five times does not influence the optimal results.

\section{Optimized Results and Discussions}

As mentioned in the previous sections that there are several explicit correlation models have been developed to estimate the value of the friction factor in the turbulent flow regime as an alternative to Colebrook equation. It would, therefore, be worthwhile to explore the accuracy of these models as compared with Colebrook equation. As a consequence, during the present study, results have been obtained for different $R e$ and $\varepsilon / D$ that are briefed in Fig. 2 in the form of $f_{D}$. The optimized results of $f_{D}$ in Fig. 2 display the solutions obtained from the fifth runs for all the model that considered in this study. It can be observed from Fig. 2 that the best and the mean values of Model-1 and Model-5 are nearly identical with Colebrook equation. The comparison between the results of $f_{D}$ that obtained by applying single-objective optimization is presented in Fig. 3 and Table 1. It is evident that the Model-1 and Model-5 provide the lowest percentage of difference with Colebrook equation which is equal to 0.05 and then, the remaining models have a percentage of difference up to 0.11. Most importantly, the results show that the GAs has been succeeded for eliminating the iterative process and reduces the computational time through estimating the $f_{D}$. Therefore, it is recommended in the future while determining the exact solution of $f_{D}$ along with either Model-1 or Model-5. 

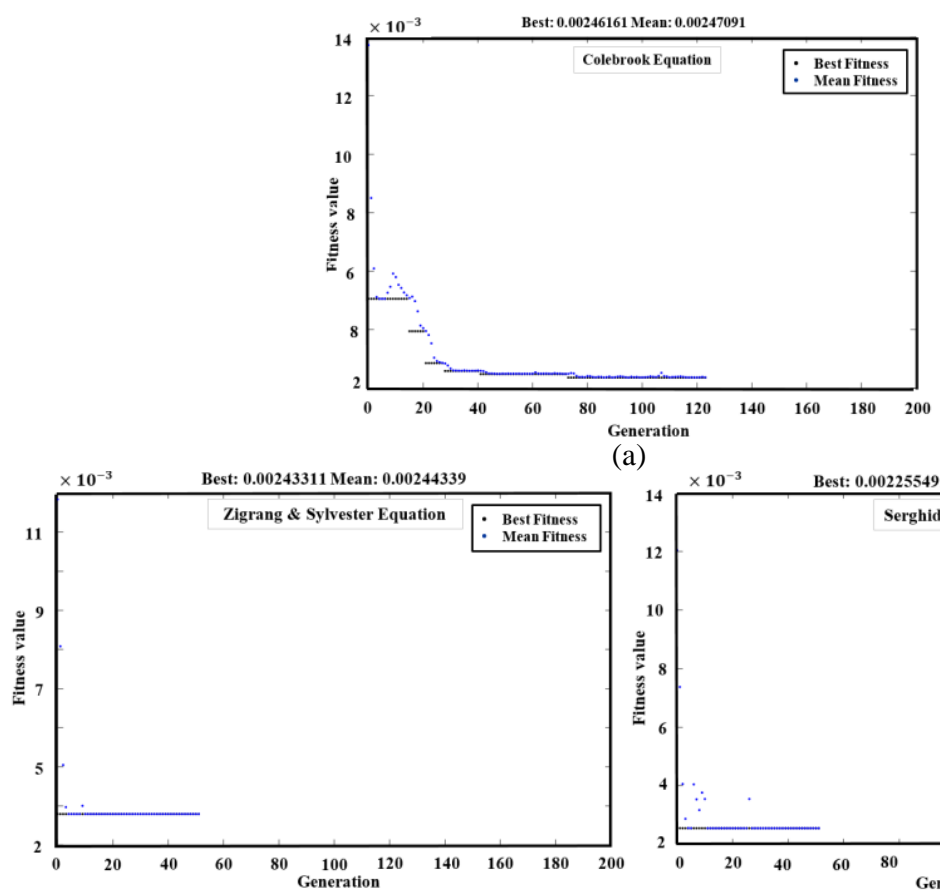

(a)

(b)
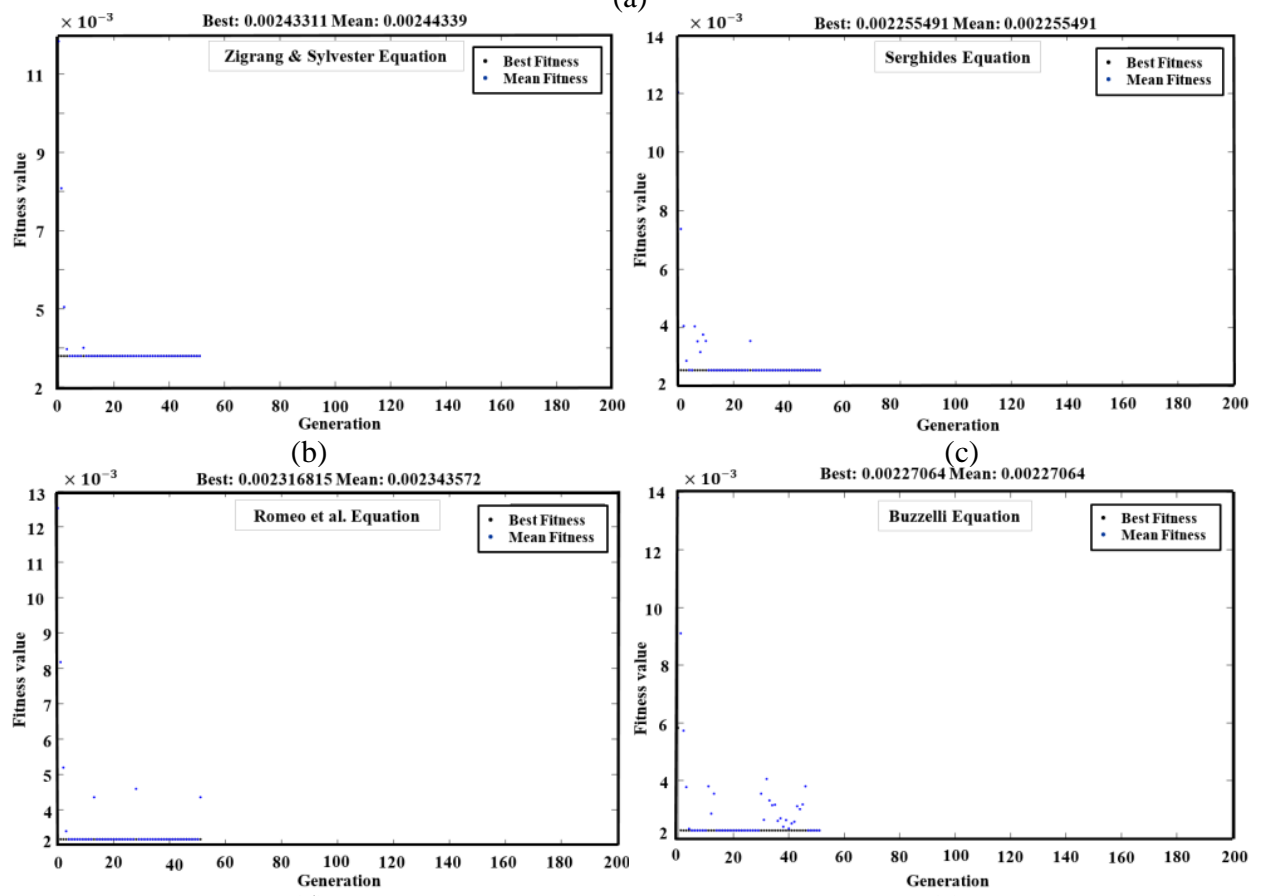

(d)

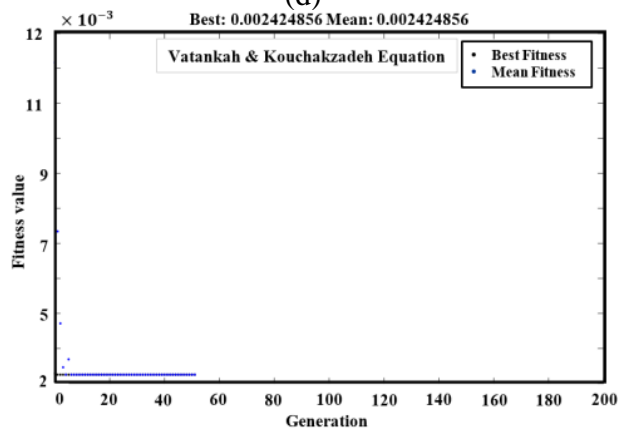

(f)

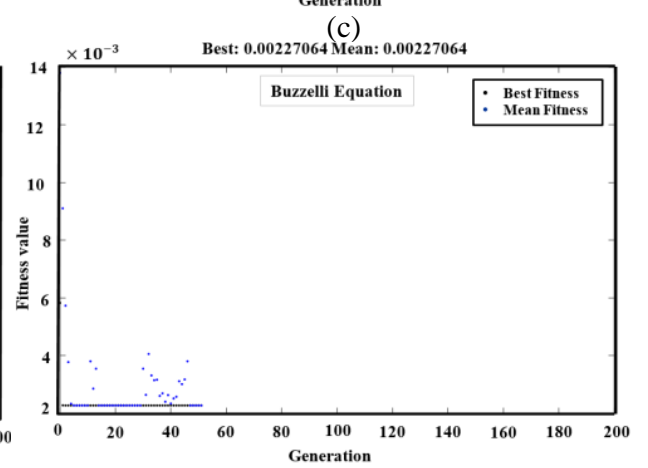

(e)

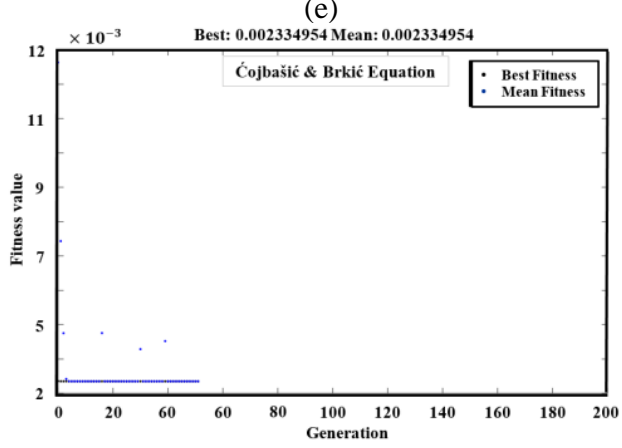

(g)

Fig. 2 The fifth single objective optimization runs for the correlation of (a) Colebrook, (b) Model-2, (c) Model-2, (d) Model-3, (e) Model-4, (f) Model-5 and (g) Model-6 


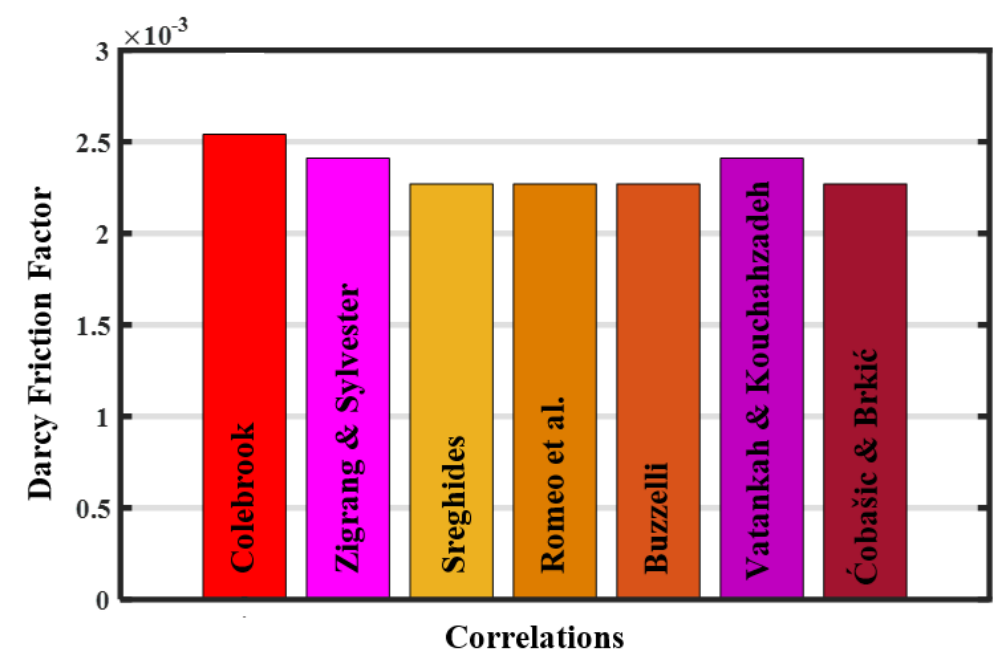

Fig. 3 Comparison between the optimal results obtained from the six explicit correlations and Colebrook equation

Table 1. Percentage of the difference between the optimal results obtained from the six explicit correlations and Colebrook equation

\begin{tabular}{lccc}
\hline Models & $\begin{array}{c}f_{D} \text { value from } \\
\text { explicit correlation }\end{array}$ & $\begin{array}{c}f_{D} \text { value from } \\
\text { Colebrook Equation }\end{array}$ & $\begin{array}{c}\text { Percentage of } \\
\text { Difference }\end{array}$ \\
\hline Zigrang and Sylvester & 0.00241 & & 0.05 \\
Serghides & 0.00227 & & 0.11 \\
Romeo et al. & 0.00227 & 0.00254 & 0.11 \\
Buzzelli & 0.00227 & & 0.11 \\
Vatankah and Kouchahzadeh & 0.00241 & 0.05 \\
Ćojbaší and Brkić & 0.00227 & 0.11 \\
\hline
\end{tabular}

\section{Conclusions}

The present research article involves a numerical study on the evaluation of the explicit correlations as alternatives to the implicit Colebrook equation for estimating the Darcy friction factor $\left(f_{D}\right)$ in a turbulent flow regime. Six explicit correlation models have been considered and these correlations have been expressed in terms of Reynolds number $(R e)$ and relative roughness $(\varepsilon / D)$. The single objective optimization along with Genetic Algorithms (GAs) has been used to obtain a solution for each correlation. This algorithm has been used to reduce the 
computational time by avoiding the iteration process. Based on this study, the following conclusions are drawn:

1. The results show that the Models 1 and 5 provide the lowest difference of the optimized $f_{D}$ with Colebrook equation under the same range of $R e$ and $\varepsilon / D$. The maximum percentage of difference has been found equal to 0.05 for Model- 1 and Model-5, whereas the remaining explicit models have been found up to 0.11 .

2. The results of the optimization process show that GAs has conformity in explanation these different equations with the important solutions. This is helpful for estimating $f_{D}$ owing to these equations can be tended to give more than one solution at a certain value of $R e$ and $\varepsilon / D$.

3. Finally, this study shows that the proposed GAs method succeeded in eliminating the iterative process and therefore, it is recommended along with either Model-1 or Model-5 in the future for finding the precise solution of the $f_{D}$.

\section{References}

[1] C. F. Colebrook, C. M. White, Experiments with fluid friction in roughened pipes. Proceedings of the Royal Society A: Mathematical, Physical and Engineering Sciences, 161 (906) (1937) 367 - 381.

[2] D. Clamond, Efficient resolution of the Colebrook equation, Industrial and Engineering Chemistry Research, 48 (7) (2009) 3665 - 3671.

[3] L. Gosselin, M. Tye-Gingras, F. Mathieu-Potvin, Review of utilization of genetic algorithms in heat transfer problems, International Journal of Heat and Mass Transfer, 52 (2009) $2169-2188$.

[4] N. Queipo, R. Devarakonda, J. A. C. Humphrey, Genetic algorithms for thermosciences research application to the optimized cooling of electronic components, International Journal of Heat and Mass Transfer, 37 (6) (1994) $893-908$.

[5] T. T. Chow, G. Q. Zhang, Z. Lin, C. L. Song, Global optimization of absorption chiller system by genetic algorithm and neural network, Energy and Buildings, 34 (1) (2002) 103 - 109.

[6] G. N. Xie, Q. Wang, B. Sunden, Application of a Genetic Algorithm for Thermal Design of Fin-andTube Heat Exchangers, Heat Transfer Engineering, 29 (7) (2008) 597 - 607.

[7] G. N. Xie, B. Sunden, Q. Wang, Optimization of compact heat exchangers by a genetic algorithm, Applied Thermal Engineering 28 (2008) 895 - 906.

[8] M. Özger, G. Yildirim, Determining turbulent flow friction coefficient using adaptive neuro-fuzzy computing technique, Advances in Engineering Software, 40 (4) (2009) 281 - 287.

[9] D. Brkić, An explicit approximation of Colebrook's equation for fluid flow friction factor, Petroleum Science and Technology, 29 (2011) 1596 - 1602.

[10] D. Brkić, Discussion of "Gene expression programming analysis of implicit Colebrook-White equation in turbulent flow friction factor calculation" by Saeed Samadianfard [ J. Pet. Sci. Eng. 92 93 (2012) 48-55], Journal of Petroleum Science and Engineering, 124 (2012) 399 - 401.

[11] S. M. Besarati, P. D. Myers, D. C. Covey, A. Jamali, Modeling friction factor in pipeline flow using a GMDH-type neural network, Cogent Engineering, 2 (2015) 1056929.

[12] C-M. Lin, H-Y. Liu, K-Y. Tseng, S-F. Lin, Heating, ventilation, and air conditioning system optimization control strategy involving fan coil unit temperature control, Applied Scinces, 9 (2019) 2391. 
[13] P. Wildi-Tremblay, L. Gosselin, Minimizing shell-and-tube heat exchanger cost with genetic algorithms and considering maintenance, International Journal of Energy Research 31 (9) (2007) $867-885$.

[14] M. G. Normah, A. Y. Qais, S. P. Agus, N. Sentot, A. Robiah, Optimization of the friction factor and frictional pressure drop of R22 and R290, International Journal of Technology 2 (2016) 227 - 234.

[15] A. Y. Qais, M. G. Normah, Z. Nor Atiqah, N. Sentot, S. P. Agus, A. Robiah, Modeling of the minimized two-phase flow frictional pressure drop in a small tube with different correlations. Journal Teknologi (Sciences \& Engineering) 78 (2016) 109-115.

[16] A. Y. Qais, M.G. Normah, S. P. Agus, Analysis of outcomes of the frictional pressure drop prediction using different data sources, Journal Teknologi (Sciences \& Engineering, 78 (10-2) (2016) 29-37.

[17] M.G.Normah, S.P. Agus, J.-T. Oh, A. Robiah, K. F. Muhammad, Rabin, Effects of two-phase flow friction factor correlations on the optimal pressure drop-martinelli parameter pair in a mini-channel, Applied Mechanics and Materials, 819 (2016) 309 - 313.

[18] M.G. Normah, J.-T. Oh, A. Robiah, Z. Nor Atikah, Optimal frictional pressure drop and vapor quality relationship of ammonia and R22 in two-phase flow, Applied Mechanics and Materials, 819 (2016) $314-318$.

[19] D. J. Zigrang, N. D. Sylvester, Explicit approximations to the solution of Colebrook's friction factor equation, AIChE Journal, 28 (3) (1982) $514-515$.

[20] T. K. Serghides, Estimate friction factor accurately, Chemical Engineering, 91 (5) (1984) 63-64.

[21] E. Romeo, C. Royo, A. Monz'on, Improved explicit equations for estimation of the friction factor in rough and smooth pipes, Chemical Engineering Journal, 86 (3) (2002) 369-374.

[22] D. Buzzelli, Calculating friction in one step. Machine Design, 80 (12) (2008) 54 - 55.

[23] A. R. Vatankhah, S. Kouchakzadeh, Discussion of "turbulent flow friction factor calculation using a mathematically exact alternative to the colebrook-white equation" by Jagadeesh R. Sonnad and Chetan T. Goudar, Journal of Hydraulic Engineering, 134 (8\#9 (2008) 1187.

[24] Ž. Ćojbašić, D. Brkić, Very accurate explicit approximations for calculation of the Colebrook friction factor, International Journal of Mechanical Sciences, 67 (2013) 10 - 13.

[25] MATLAB R2018a Version (9.4.0.813654) 64 Bit (win64), License Number 968398. 